\title{
Modified branched reverse frozen elephant trunk repair for failed TEVAR
}

\author{
Shinichi Fukuhara, Eric E. Roselli \\ Aorta Center, Department of Thoracic and Cardiovascular Surgery, Heart and Vascular Institute, Cleveland Clinic, Cleveland, OH, USA \\ Correspondence to: Eric E. Roselli, MD. Department of Thoracic and Cardiovascular Surgery, Cleveland Clinic, 9500 Euclid Avenue, Desk J4-1, \\ Cleveland, OH 44915, USA. Email: ROSELLE@ccf.org.
}

Submitted May 05, 2018. Accepted for publication May 16, 2018.

doi: 10.21037/acs.2018.05.09

View this article at: http://dx.doi.org/10.21037/acs.2018.05.09

\section{Clinical vignette}

A 73-year-old asymptomatic primary care physician was referred to our Aorta Center with aortic arch and root aneurysms, and a chronic distal aortic dissection. The acute type B aortic dissection occurred two years earlier and was complicated by mesenteric ischemia requiring an emergency thoracic endovascular aortic repair (TEVAR) using a $45 \mathrm{~mm} \times 150 \mathrm{~mm}$ stent graft device (CTAG, WL Gore and Associates, Flagstaff, AZ, USA) performed at an outside institution (Figure 1). He tolerated the procedure well with resolution of the ischemia and expansion of the true lumen. The postoperative course was uneventful, but he had persistent degeneration and growth of his aortic arch proximal to the stent-graft and through the descending aorta.

He had several follow-up computed tomography (CT) scans that showed a type $1 \mathrm{~A}$ endoleak originating in the arch and an associated "bird-beak" configuration of the stent-graft. The aortic arch aneurysm proximal to the endograft had also been slowly growing, measuring $6.2 \mathrm{~cm} \times$ $6.0 \mathrm{~cm}$ in diameter (Figure 2). The residual aortic dissection extended from the level of the left subclavian artery into the left external iliac artery. The size of the dissected middescending aorta measured $5.2 \mathrm{~cm} \times 4.6 \mathrm{~cm}$ and has been relatively stable. His aortic root was also dilated measuring $4.5 \mathrm{~cm}$. Transthoracic echocardiography demonstrated a tricuspid aortic valve with mild aortic insufficiency and normal biventricular function. In preparation for surgical repair, he underwent coronary angiography that was unremarkable. He was recommended to undergo elective aortic root, ascending and total arch replacement with a modified branched frozen elephant trunk repair to include stent grafting into the left subclavian and left carotid arteries. It was also explained that this may be the first of a staged repair approach to his extensive dissection disease.

\section{Surgical technique}

We have been performing extended repair for acute DeBakey type 1 aortic dissection and chronic type B dissection involving aortic arch and descending aorta pathologies using a hybrid simplified frozen elephant trunk strategy for several years (1-3). This technique is particularly useful in patients with retrograde dissection from a distal entry tear, aneurysmal changes in the more distal aorta, distal malperfusion and those with a connective tissue disorder or family history of aortic disease placing them at increased risk for false lumen aneurysm of the downstream aorta in the presence of residual dissection (4). This surgical technique has evolved over the last 7 years and the latest method for patients undergoing elective repair is demonstrated in the accompanying video.

\section{Preparation}

Careful review of contrast enhanced CT imaging using three-dimensional reconstruction software is the most critical step in the preparation for the procedure. Accurate measurements of the aorta at the level between the left common carotid and left subclavian arteries (zone 2), and each of the supraaortic branch vessels in a plane orthogonal to the center line of flow were performed. Stent-graft devices used for the head vessels and the aorta were preselected based on this analysis with minimal oversizing to avoid endograft folding or gutter formation. For the main 

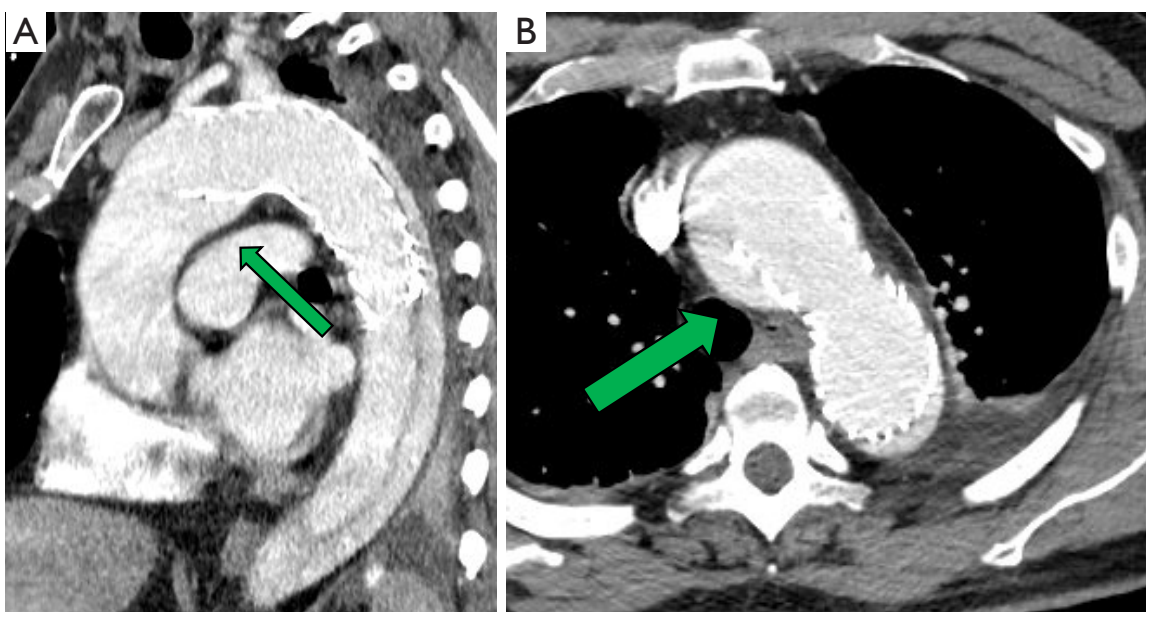

Figure 1 Multi-planar reconstruction of contrast enhanced computed tomography performed after his initial TEVAR repair in the sagittal (A) and axial (B) planes. Green arrow in the sagittal view demonstrates the so-called "bird beak" mal-apposition of the device to the aortic wall in the arch. Green arrow in the axial view demonstrates the dilated proximal arch. TEVAR, thoracic endovascular aortic repair.
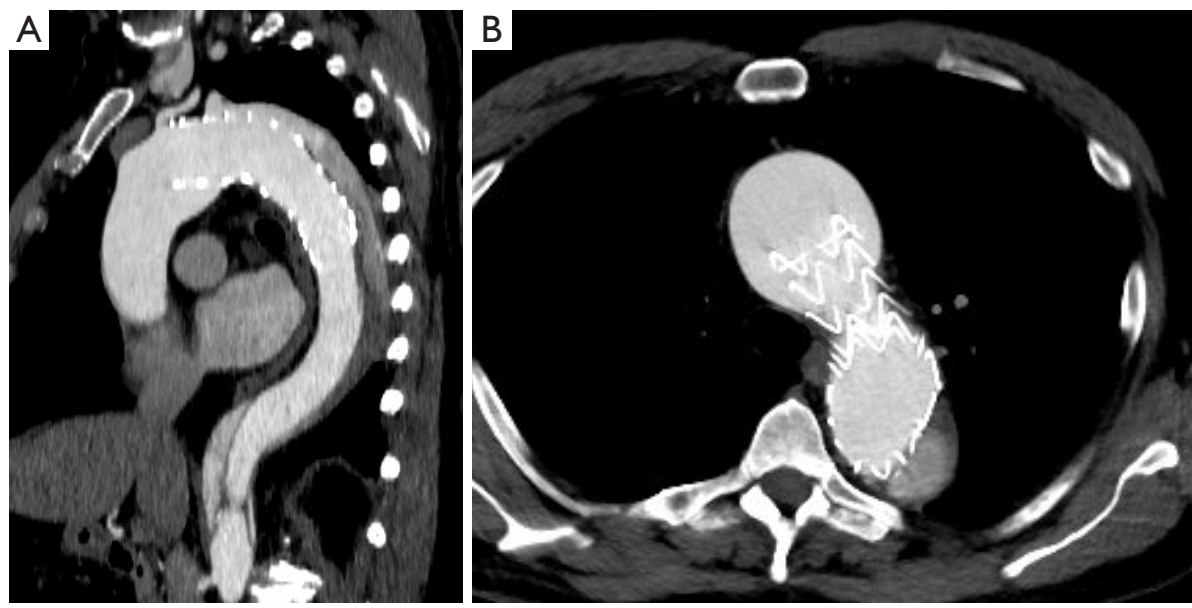

Figure 2 Multi-planar reconstruction of contrast enhanced computed tomography performed 2 years after his initial TEVAR repair in the sagittal (A) and axial (B) planes demonstrated the mal-apposed stent in the arch with surrounding endoleak and aneurysmal proximal arch. TEVAR, thoracic endovascular aortic repair.

aortic stent-graft, the length of the device is usually 10 to $15 \mathrm{~cm}$ depending on the configuration of the aortic arch. In general, it should at least extend around the curve of the arch and into the proximal descending aorta. A $2.5 \mathrm{~cm}$ length device is preferred for devices for the left subclavian artery to prevent coverage of the left vertebral artery and limit the potential for injury to a commonly tortuous vessel. Cerebrospinal fluid drainage is a useful tool to prevent spinal cord injury and is usually placed in patients undergoing elective frozen elephant trunk procedure for chronic dissection. An arterial line can be placed into either upper extremity. A large bore right internal jugular catheter with a pulmonary artery catheter was placed following standard induction for general anesthesia. Broadspectrum intravenous antibiotics with both gram positive and negative coverage were administered. Transesophageal echocardiography (TEE) was performed to assess valve function, aortic annulus diameter and biventricular function. There was a posteriorly directed regurgitant jet likely secondary to right coronary cusp prolapse. Cerebral 
monitoring was facilitated by the use near infrared spectroscopy (NIRS) and bispectral index (BIS) monitor.

\section{Exposure}

Standard median sternotomy was performed and systemic heparin was administered. CPB was established through central aortic cannulation at level of the distal ascending aorta where the aorta was not excessively aneurysmal and standard right atrial cannulation using a multi-stage cannula. The coronary sinus was cannulated for the delivery of retrograde cardioplegia. A self-retaining retractor (Hercules; Estech, West Chester, OH, USA) is attached to the sternal retractor to retract the innominate vein upward and away from the supra-aortic branch vessels. The aortic arch was adequately mobilized. The innominate artery and left common carotid artery were encircled with vessel loops for preparation of selective antegrade brain perfusion (SABP).

\section{Operation}

Cooling was initiated to a goal blood temperature of $18-20^{\circ} \mathrm{C}$, and the head was packed in ice. BIS monitor was used to confirm adequate cooling. The aorta was crossclamped and antegrade and retrograde cardioplegia was given to arrest the heart. The aorta was transected at the level of the sinotubular junction. His aortic root was moderately dilated with annuloaortic ectasia. The aortic valve had some degenerative changes along the stress zones of the right and non-coronary cusps with fenestrations but the rest of the leaflets looked good. A decision was made to proceed with a valve-sparing aortic root reimplantation. Both coronary buttons were isolated and mobilized, and the aortic root was mobilized well below the aorto-ventricular junction. Subannulatus interrupted pledgeted 2-0 braided sutures were placed under each of the commissures and the nadir of each leaflet. At this point, the target temperature was achieved with confirmation of BIS silence. The aortic crossclamp was removed and flow was reduced to $500 \mathrm{cc} / \mathrm{min}$ while the innominate and left common carotid artery were individually cannulated for SABP. Brain flow was maintained at a rate of about $800-1,000 \mathrm{cc} / \mathrm{min}$ at a perfusate temperature of $18{ }^{\circ} \mathrm{C}$. To avoid steal from the left vertebral system and eliminate back-bleeding to the surgical field, a 9-Fr occlusion balloon was placed into the left subclavian artery. The aorta was transected at level of zone 1 and the origin of the left common carotid artery was oversewn. The proximal end of the previous CTAG device was adherent but did not appear to fully seal against the aortic wall. The old stent graft device was mobilized to expose the origin of the left subclavian artery that had previously been covered. A new $45 \mathrm{~mm} \times 100 \mathrm{~mm}$ "precurved" CTAG device was delivered under direct vision into the distal arch and proximal descending aorta overlapping with the old stent-graft, and was deployed in zone 1 . Its position was manually adjusted, and it was secured to the lesser curve of the aortic arch. The device was balloondilated using a $46 \mathrm{~mm}$ conformable balloon (Coda; Cook Inc, Bloomington, IN, USA). A fenestration in both the new and the old stent-grafts underneath the base of the left subclavian artery was created using a handheld eyeball cautery and a $13 \mathrm{~mm} \times 25 \mathrm{~mm}$ covered self-expanding stent (Viabahn ${ }^{\circledR}$; WL Gore and Associates) was delivered into the left subclavian artery through the fenestration (Figure 3). The fenestration and the overlapping portion of the stent were gently spread wide with a right-angle clamp. The stent was subsequently balloon-dilated using the 9-Fr occlusion balloon to gain adequate seal. Its positioning was confirmed by external palpation of the left subclavian artery as well as visually inside the aortic device. The occlusion balloon was left in place during the circulatory arrest. The main aortic stent-graft device was directly sutured to the transected aortic wall with a running 4-0 polypropylene suture buttressed with a strip of bovine pericardium on the outer wall. A $30 \mathrm{~mm}$ collared graft (Gelweave Siena; Vascutek Terumo, Renfrewshire, Scotland) was selected based on the size of the sinotubular junction. The collar of the graft was utilized to address the size discrepancy between the proximal transected aorta and the CTAG device. The collar of the surgical graft was circumferentially trimmed and sutured to the transected aorta and the edge of the stent graft altogether. After completion of this anastomosis, the distal aorta was de-aired and full CPB flow to the lower body and LSA was reinstituted. At this point, the hemostasis of the pressurized suture line was thoroughly assessed and repair stitches were placed as needed. The most distal unused $8 \mathrm{~mm}$ side branch of the graft was ligated and cut. Next, the left common carotid artery and an $8 \mathrm{~mm}$ side branch was aligned and trimmed to an appropriate length. The two were secured with a 5-0 figure-of-eight suture on the posterior wall. A $10 \mathrm{~mm} \times 50 \mathrm{~mm}$ Viabahn was delivered through the $8 \mathrm{~mm}$ graft into the left carotid artery and deployed to create a quick hybrid anastomosis. Two additional 5-0 Prolene sutures were placed through the carotid artery, $8 \mathrm{~mm}$ graft and the stent-graft to secure the device. The base of the innominate artery was then 

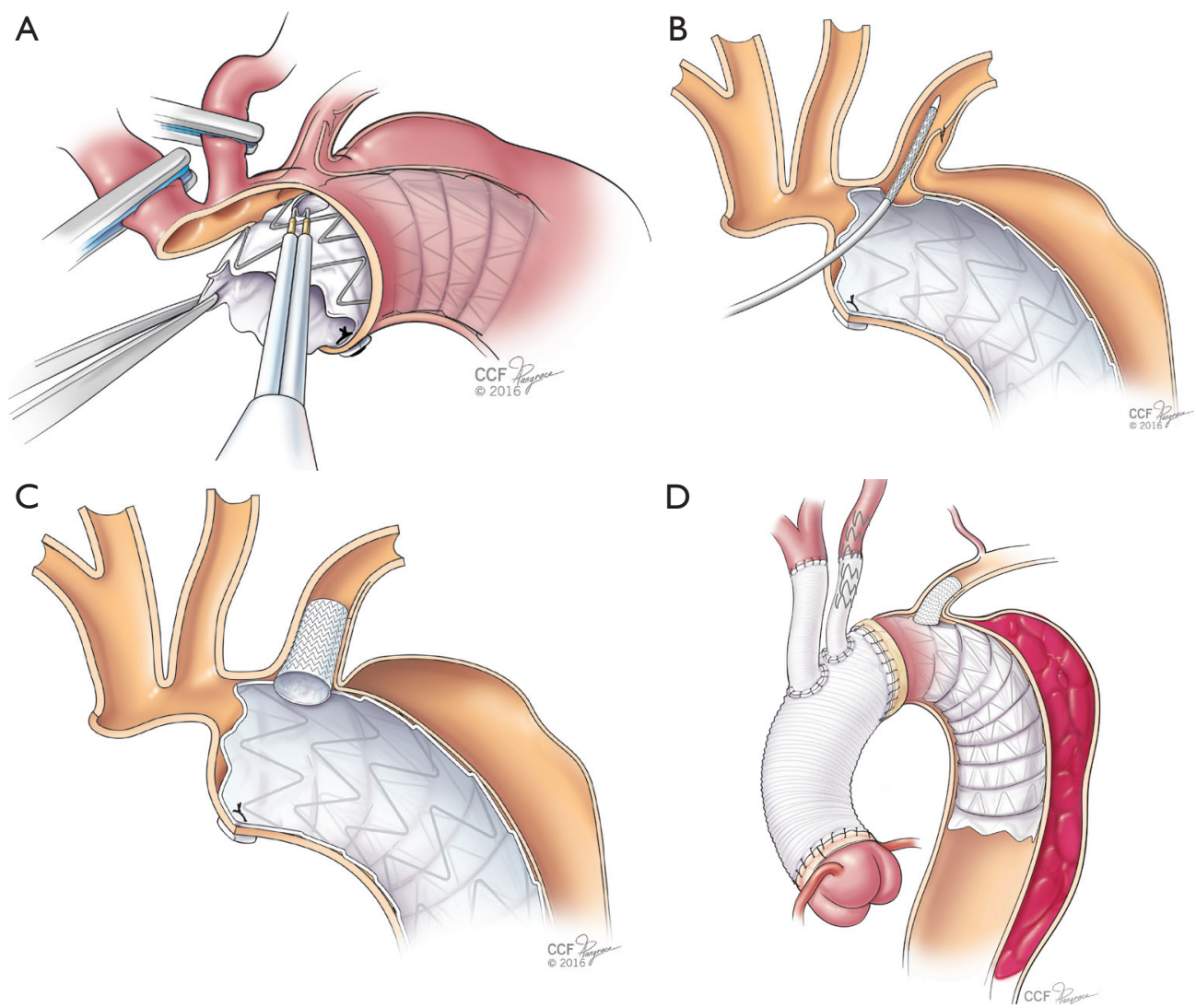

Figure 3 Illustrations demonstrating some of the steps of performing the reverse frozen elephant trunk repair with modified branching. (A) Demonstrates the burning of the fenestration into the stent graft, followed by branch device delivery with a smaller covered self-expanding covered stent in (B). The device is then deployed with overlap into the aortic lumen in (C). (D) Demonstrates a completed repair with anastomosis in the mid to proximal arch zone 1 or 2 with the stented branch into the left subclavian artery across the frozen elephant trunk, hybrid anastomosis of the left common carotid artery and direct anastomosis to the innominate trunk.

anastomosed to the trimmed $10 \mathrm{~mm}$ side branch graft with a conventional running 5-0 suture line.

\section{Completion}

After correction of metabolic derangements was confirmed, the rewarming process was resumed. Attention was returned to the proximal aortic reconstruction. A $30 \mathrm{~mm}$ graft (Gelweave Valsalva; Vascutek, Terumo) was sewn to the left ventricular outflow tract. The valve was then reimplanted into the new aortic root with running 4-0 polypropylene sutures and then the left and the right coronary buttons were reimplanted in an end-to-side fashion to the new aortic root. The free margin of the right coronary cusp was shortened along the right/non-coronary commissure using a 5-0 braided suture (Ethibond, Ethicon) to equal the free margin length of the right cusp with the other cusps. This maneuver closed the fenestrations in that area as well. Finally, the graft-graft anastomosis was completed, the heart was de-aired, and the cross-clamp was removed. The CPB time was 219 minutes, SABP time was 45 minutes and the aortic cross-clamp time was 184 minutes. He was weaned from CPB without difficulty. TEE confirmed preserved biventricular function and well-functioning valves without aortic insufficiency or stenosis. The patient was taken to the ICU in stable condition.

\section{Follow-up}

A multi-phase CT demonstrated an excellent proximal repair with a well seated graft and stent grafts, patent branch vessels with good positioning, and no kinks (Figure 4). At 

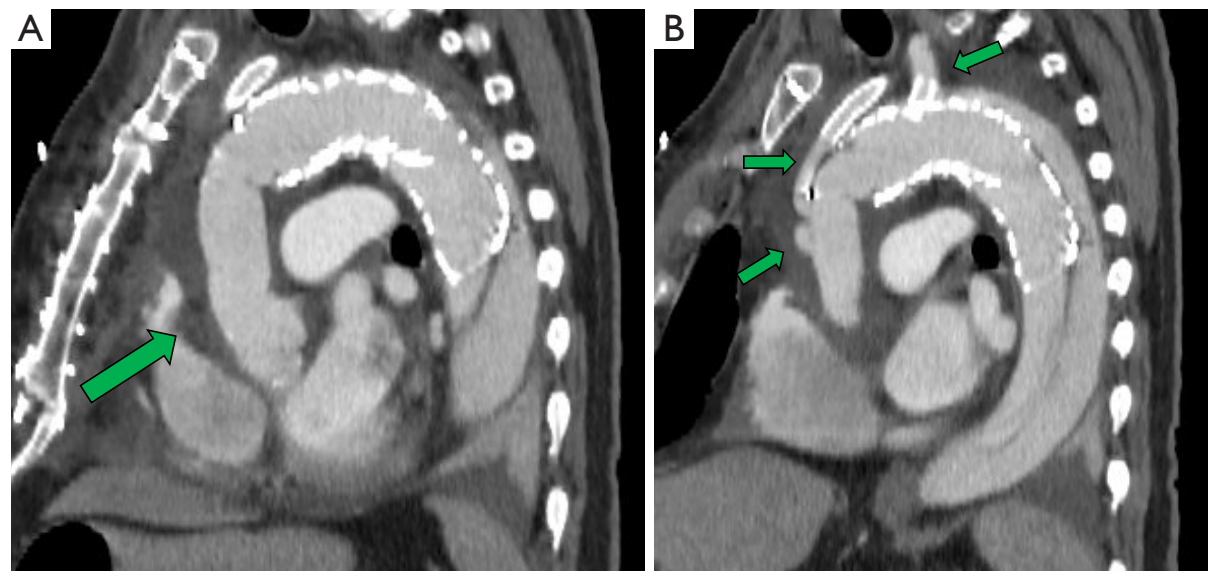

Figure 4 Contrast enhanced multi-planar reconstruction CT scan images of the thoracic aorta post repair. (A) Demonstrates the root replacement with valve reimplantation (arrow) and (B) demonstrates the patent arch branch vessels (arrows).

6 months post-operation the patient has recovered well without any sequelae. The distal residually dissected aorta still demonstrates false lumen perfusion with a maximum diameter of $48 \mathrm{~mm}$ beyond the repair. This will likely require a second stage endovascular elephant trunk completion procedure at a later date (4).

\section{Comments}

TEVAR for patients with acute complicated type 3 dissection has been shown to improve aorta-specific survival with less late complications compared to medical management alone (5). Many survivors are subject to aortic complications including distal aortic dilatation, aneurysmal formation, malperfusion syndrome and potential rupture. The management of extensive aortic disease including aneurysm and dissection often requires a staged approach and often times, repeated surgical interventions $(6,7)$. Increasingly, this also includes the use of both open and endovascular approaches, as demonstrated in the present video. Aneurysmal degeneration of the aorta in patients with chronic dissection commonly occurs in the distal aortic arch and proximal descending aorta, making the frozen elephant trunk technique well-suited for this indication (8). When the patient's disease demands a descending TEVAR repair first, a frozen elephant trunk strategy is still very successful in a reverse frozen elephant trunk fashion (revFET).

\section{Clinical results}

We have performed this modified branched frozen elephant trunk repair in many patients since 2015 for acute and chronic type 3 dissection, degenerative aneurysm, and acute DeBakey type 1 dissection with consistent and complete technical success in all patients and no intraoperative deaths. The updated clinical results for the whole cohort are to be presented and published elsewhere in the near future, but we have recently published our experience in those undergoing this type of repair for acute DeBakey type 1 dissection (3). Our follow-up protocol includes clinical and CT imaging assessment performed prior to discharge, within the first 3 to 6 months, then at 12 months postoperatively, and annually thereafter. We consider all patients with thoracic aortic disease to have a life-time risk for additional disease, and the open and endovascular approaches to repair as complementary to each other.

\section{Advantages}

One of the advantages of this technique is its versatility. It can be tailored to the specific anatomical and morphological aspects of the patient's disease process with the in-situ creation of a fenestration in the aortic stent-graft and the direct placement of covered stents into the left subclavian artery (1-3). Our techniques have further evolved to include additional hybrid approaches to anastomosis arch branch vessels with covered stents as was done for the left carotid artery in this patient. By bringing the distal aortic anastomosis closer to the surgeon hemostasis may be improved and injury to the recurrent laryngeal nerve injury is less likely. In some patients, this particular technique can also be applied to the innominate artery-branch graft 
anastomosis. Utilization of this modification can shorten the circulatory arrest time during aortic arch reconstruction procedures, a factor that has been frequently correlated with neurologic risk.

\section{Caveats}

These devices are used in an off-label manner for this operation. Experience with, and a detailed understanding of endovascular devices is imperative in order to apply this technique. Choosing the proper size stent-graft devices is always critical with endovascular devices but with this operation, the direct suturing of the device does allow some additional leeway. Possible procedural issues, particularly in emergency settings include stent-graft induced new entry, spinal cord injury, prolonged circulatory arrest and stroke (9). Another well-described limitation of the staged elephant trunk approach is the risk of rupture between stages. Earlier timing for the second stage TEVAR should be considered in high risk patients and additional techniques like open fenestration during the first stage elephant trunk can improve the applicability of second stage TEVAR (10). Further evaluation of this and other variations of hybrid arch reconstructions techniques is ongoing and will improve our understanding of how to best tailor individual patient therapy.

\section{Acknowledgements}

None.

\section{Footnote}

Conflicts of Interest: EE Roselli is a consultant and investigator for Terumo Aortic, Cook, Gore, and Medtronic. S Fukuhara has no conflicts of interest to declare.

\section{References}

1. Roselli EE, Rafael A, Soltesz EG, et al. Simplified frozen elephant trunk repair for acute DeBakey type I dissection. J Thorac Cardiovasc Surg 2013;145:S197-201.
2. Roselli EE, Tong MZ, Bakaeen FG. Frozen elephant trunk for DeBakey type 1 dissection: the Cleveland Clinic technique. Ann Cardiothorac Surg 2016;5:251-5.

3. Roselli EE, Idrees JJ, Bakaeen FG, et al. Evolution of Simplified Frozen Elephant Trunk Repair for Acute DeBakey Type I Dissection: Midterm Outcomes. Ann Thorac Surg 2018;105:749-55.

4. Roselli EE, Subramanian S, Sun Z, et al. Endovascular versus open elephant trunk completion for extensive aortic disease. J Thorac Cardiovasc Surg 2013;146:1408-16; discussion 1416-7.

5. Shrestha M, Bachet J, Bavaria J, et al. Current status and recommendations for use of the frozen elephant trunk technique: a position paper by the Vascular Domain of EACTS. Eur J Cardiothorac Surg 2015;47:759-69.

6. Nienaber CA, Kische S, Rousseau H, et al. Endovascular repair of type $\mathrm{B}$ aortic dissection: long-term results of the randomized investigation of stent grafts in aortic dissection trial. Circ Cardiovasc Interv 2013;6:407-16.

7. Zeeshan A, Woo EY, Bavaria JE, et al. Thoracic endovascular aortic repair for acute complicated type B aortic dissection: superiority relative to conventional open surgical and medical therapy. J Thorac Cardiovasc Surg 2010;140:S109-15; discussion S142-6.

8. Roselli EE, Bakaeen FG, Johnston DR, et al. Role of the frozen elephant trunk procedure for chronic aortic dissection. Eur J Cardiothorac Surg 2017;51:i35-9.

9. Katayama K, Uchida N, Katayama A, et al. Multiple factors predict the risk of spinal cord injury after the frozen elephant trunk technique for extended thoracic aortic disease. Eur J Cardiothorac Surg 2015;47:616-20.

10. Aftab M, Idrees JJ, Cikach F, et al. Open Distal Fenestration of Chronic Dissection Facilitates Endovascular Elephant Trunk Completion: Late Outcomes. Ann Thorac Surg 2017;104:1960-7.

Cite this article as: Fukuhara S, Roselli EE. Modified branched reverse frozen elephant trunk repair for failed TEVAR. Ann Cardiothorac Surg 2018;7(3):437-442. doi: 10.21037/acs.2018.05.09 